\title{
Noncompliance in survival surgery technique
}

Great Eastern University's surgical training program covered everybody who performed major survival surgery for research purposes, including board-certified surgeons, veterinarians, technicians and others. People who claimed to have basic surgical competency were thoroughly tested in aseptic technique and basic surgical skills by a surgical trainer, using a simulated animal. Basic skill training was provided by a surgical trainer for those who required it. Advanced training for specialized techniques was provided by the surgical trainer or the research laboratory, using previously euthanized animals and progressing to nonsurvival surgery, as specified in the IACUC protocol. A veterinarian or training specialist always observed the first one or two survival procedures; if there were perceived problems, the trainer was required to immediately inform the Attending Veterinarian or IACUC chairperson.

The training and testing program worked almost flawlessly, until the veterinarians received a report from a veterinary technician that animals on which Linda Girard, a postdoctoral fellow, had operated two days earlier had a high incidence of wound dehiscence and infection. Some had to be euthanized. Necropsies revealed that the surgical procedure had not been carried out well. When confronted, Girard admitted that she took protocol 'shortcuts' because she was overwhelmed with lab work. The shortcuts included wiping instruments with alcohol rather than sterilizing them between procedures on different animals when multiple animals were operated on during the same surgical session. She disinfected but did not change her surgical gloves between procedures on different animals. She used a continuous suture pattern to close the abdominal wound when individual sutures were specified. Lastly, she sutured the peritoneum, muscle and skin as a unit, rather than closing the skin incision independently from the other layers. All of these shortcuts were contrary to her IACUC training. The findings were promptly transmitted to the IACUC, and the committee immediately convened an emergency meeting with Girard and the Principal Investigator (PI) in attendance. After a discussion, the committee found itself in a quandary. Girard had been properly trained, and her surgical technique had been observed and approved. The current problem had been quickly identified. When Girard and the PI were told by the IACUC what was required to bring the study back into compliance, they immediately agreed to do it. Additional IACUC monitoring of Girard's work was mandated. But was the infraction of sufficient magnitude for Girard to be suspended? Even if she were not suspended, should this incident be reported to the Office of Laboratory Animal Welfare at the National Institutes of Health? Some IACUC members said yes to the suspension and report, while others disagreed. Those that disagreed said that in order for the committee to suspend Girard or report the incident, the details of the committee's expectations (e.g., changing surgical gloves between animals) should have been specified in the approved protocol or at least be part of a written IACUC policy. Because neither of these conditions was met, they believed that Girard could not be tightly bound to a nonexistent policy or nonexistent protocol details.

What do you think the Great Eastern IACUC should do?

\section{RESPONSE}

\section{Academic misconduct}

\section{Stephen M. Dempsey, DVM \& \\ Judith R. Lassiter, BA, CPIA}

We congratulate Great Eastern University on its surgical training program. The fact that this case is an exception to an otherwise flawless record is a testament to the quality of the training that its faculty and staff receive.

There are two main issues in Girard's case: workload and academic (dis)honesty. There is no excuse for taking shortcuts because of other lab work; it is incumbent upon Girard, as a postdoctoral fellow, to discuss her workload with the Principal Investigator (PI) and to determine what measures could be taken to prioritize or even reassign some of her duties to allow her adequate time to carry out the surgical procedures in accordance with the protocol and Great Eastern's accepted aseptic surgery standards. The PI, who presumably signed some form of an assurance statement attesting to the training, competence and performance of individuals who carry out animal activities on his or her protocol, also bears responsibility to mentor and oversee the activities of everyone who is assigned animal activities under his or her protocol and to assist the graduate and postdoctoral trainees in becoming responsible PIs themselves.

There has been a substantial uptick in the numbers of cases of academic misconduct in many universities. Some may not see Girard's shortcuts as academic dishonesty, but these actions are really no different from falsifying scientific data or plagiarizing information, just less public. In this case, Girard should be temporarily suspended and should undergo re-training in aseptic surgical technique. Moreover, she should also be mandated to take further training or retraining on research ethics and compliance, so that she gains a better understanding of her responsibilities, her role in the bigger picture and her future as an independent investigator. She should carry out any surgical procedures under direct supervision of a qualified individual until the IACUC and veterinary staff are satisfied that she is capable of carrying out aseptic surgery properly when unsupervised. If a second similar incident is reported, Girard's surgical privileges should be permanently revoked at Great Eastern. 